\title{
PERCORSI DELL'INFINITO NEL PENSIERO FILOSOFICO E TEOLOGICO DI DUNS SCOTO
}

\author{
Alessandro Ghisalberti*
}

SÍNTESE - Com base na metafísica dos transcendentais, quer-se mostrar a análise scotista do conceito de infinito como transcendental disjuntivo e como conceito filosófico adequado para a realidade de Deus. Pressuposição para uma função positiva do conceito de ente infinito na teologia filosófica de Scotus é, ademais, a teoria da univocidade do ente.

PALAVRAS-CHAVE - Infinito. Teologia e filosofia de Duns Scotus. Transcendentais. Univocidade do ente.
ABSTRACT - Based on Scotus's metaphysics of transcendental concepts, this essay analyses the Scotist concept of the infinite as a disjunctive transcendental and as the most proper philosophical concept for the reality of God. Decisive presupposition to a positive function of the concept of infinite being in Scotus's philosophical theology is, furthermore, the theory of the univocity of being.

KEY WORDS - Infinite. Duns Scotus's theology and philosophy. Transcendentals. Univocity of being.

Le acquisizioni più significative da parte delle ricerche svolte negli ultimi decenni sull'opera di Giovanni Duns Scoto sono relative all'analisi dei testi circa la complessa nozione di "ens infinitum" in stretta connessione con quella di "ens finitum" nella sua declinazione di contingente o di possibile.

Ne sono coinvolti la questione dell'oggetto proprio della metafisica, della univocità dell'ente, dei trascendentali disgiuntivi, insieme con la possibilità di elaborare una teologia razionale che possa accordarsi con le istanze critiche nei confronti dell'aristotelismo sollevate dai teologi e fissate nelle note tesi del Sillabo parigino del 1277.

Secondo Duns Scoto, le perfezioni del reale possono essere assolutamente semplici (es. "l'essere in quanto essere"), semplici ma non assolutamente (es. "l'essere finito"), oppure complesse (es. "animale razionale"). L'essere in quanto essere, ossia la perfezione assolutamente semplice, non esiste in concreto; l'essere è sempre "modalizzato", definito da un certo grado di perfezione o modo intrinse-

Università del Sacro Cuose/Milano

\begin{tabular}{|l|l|l|l|l|l|}
\hline VERITAS & Porto Alegre & v. 50 & n. 3 & Setembro 2005 & p. 41-50 \\
\hline
\end{tabular}


$\mathrm{co}$, da cui è indissociabile. L'essere è intelligibile in quanto essere, ma attuale solo se concepito unitamente al proprio modo.

Le perfezioni assolutamente semplici possono essere infatti distinte non solo in senso reale (due perfezioni che hanno contenuti intelligibili diversi) o in senso formale (due perfezioni diverse, anche se realmente identificate nell'esistenza concreta), ma anche dal punto di vista modale, ossia mediante il confronto tra una perfezione ed il suo modo intrinseco. D'altra parte, ogni perfezione è tale solo nella misura in cui è perfezione dell'essere, che abbraccia estensivamente tutti gli enti e ne comprende tutte le determinazioni concrete.

Secondo Scoto l'essere è univoco, quando è concepito a prescindere da tutte le determinazioni: l'essere univoco ha una comprensione illimitata e designa l'esistenza attuale di ogni ente. L'essere è analogo, quando è concepito unitamente al suo modo intrinseco (finitezza o infinità), giacché è in virtù del suo modo d'essere che ogni ente si distingue da tutti gli altri, pur avendo in comune con ciascuno l'esistenza attuale. Dunque, l'univocità fonda l'analogia, secondo cui si realizza il modo concreto di ogni essere.

Oggetto primo dell'intelletto umano è l'essere in quanto essere, l'essere in senso univoco, che ha dunque un'importanza fondamentale. Infatti, comprendendo tutti gli enti (finiti e infinito), fonda l'oggettività del conoscere, senza configurarsi come un oggetto. Possiamo affermare che qualcosa è, anche senza sapere se è sostanza o accidente, finito o infinito. L'essere univoco e le determinazioni ultime sono concetti irriducibili, che stanno tra loro in relazione di potenza ed atto: l'essere univoco sta alle differenze come ciò che è ultimativamente determinabile sta a ciò che è ultimativamente determinante negli esseri.

L'essere è predicato "quidditative" di tutto quello che può essere ridotto ad un ultimo elemento determinabile, ossia al concetto comune di essere: la predicazione in "quid" avviene attraverso i generi, le specie, gli individui, tutte le parti essenziali dei generi, l'Essere increato.

L'essere è predicato "qualitative" delle differenze ultime che sono assolutamente semplici. Tale diversità di predicazione è dovuta al fatto che le differenze ultime o elementi determinanti non hanno niente in comune con il concetto univoco di essere determinabile:

Ille conceptus "tantum determinabilis" est conceptus entis, et "determinans tantum" est conceptus ultimae differentiae. Ergo isti erunt primo diversi, ita quod unum nihil includet alterius. ${ }^{1}$

L'analogia dell'essere, valida per le categorie, è molto importante per il metafisico ed il filosofo naturale, i quali sono interessati all'ordine naturale delle cose. Nel suo scritto In librum Praedicamentorum, Scoto sostiene che ci deve essere unità tra le categorie: a ciascuna infatti è attribuibile l'essere, in quanto tutte trovano il fondamento nella sostanza. Parimenti, una scienza può trarre il suo soggetto da un'unica sostanza, che è prima rispetto a tutti i soggetti specifici delle pro-

Duns Scoto, Ordinatio I, d. 3, p. 1, q. 3; ed. Vat. III, pp. 82-83, n. 133. 
posizioni vere che costituiscono il suo discorso e che può essere attribuita a ciascuno di essi. Dunque, l'unità analogica è sufficiente ad assicurare l'unità della scienza.

Nell'Ordinatio il Dottor Sottile modifica la propria posizione, sostenendo che la nozione dell'analogia dell'essere non è sufficiente per costruire una teologia razionale. Infatti, per conoscere una categoria di essere divino "analogo" all'essere creato bisognerebbe possedere o un'idea di essere assolutamente semplice su cui fondare l'analogia, o due concetti di essere - uno divino e l'altro creato - ugualmente indipendenti. ${ }^{2}$ Quando una realtà è conosciuta con il suo modo intrinseco, il suo concetto non è così assolutamente semplice da consentire di concepire la realtà stessa senza il suo modo, e perciò abbiamo un concetto imperfetto. Solo se si concepisce la realtà senza il modo, il concetto della cosa è perfetto:

Quando intelligitur aliqua realitas cum modo suo intrinseco, ille conceptus non est ita simpliciter simplex quin possit concipi illa realitas absque modo illo, sed tunc est conceptus imperfectus illius rei; potest etiam concipi sub illo modo, sed tunc est conceptus perfectus illius rei [...]. Requiritur ergo distinctio, inter illud a quo accipitur conceptus communis et inter illud a quo accipitur conceptus proprius, non ut distinctio realitatis et realitatis sed ut distinctio realitatis et modi proprii et intrinseci eiusdem, quae distinctio sufficit ad habendum conceptum perfectum vel imperfectum de eodem, quorum imperfectus sit communis et perfectus sit proprius. ${ }^{3}$

Il concetto imperfetto ma comune è il concetto univoco, il concetto di essere che può essere predicato di Dio e delle creature: "[...] univocum conceptum dico, qui ita est unus quod eius unitas sufficit ad contradictionem, affirmando et negando ipsum de eodem", "il concetto perfetto ma meno comune è un concetto più proprio dell'essere, comprendente le separate categorie. Se applicato in modo trascendentale, esso necessariamente implicherà equivocazione.

\section{Dall'ente in quanto ente all'ente infinito}

Criticando i suoi predecessori seguaci dell'agostinismo gnoseologico, Scoto ritiene incontrovertibile la posizione aristotelica secondo cui la conoscenza umana, almeno in questo mondo, attinge esclusivamente da oggetti materiali. L'unica opzione consentita è che la mente umana possa avviare il processo conoscitivo con una nozione di essere tratta dalla sua conoscenza degli enti del mondo, ma che possa essere applicata, in quanto nozione sufficientemente astratta, ad un oggetto sovrasensibile. La nozione di essere infinito, astratta dalla conoscenza delle creature, è idonea a rappresentare l'essere divino, sia pure in modo imperfetto.

Oltre a constatare la non ripugnanza dell'infinità all'ente, Duns Scoto osserva che l'infinito

\footnotetext{
Cfr. Ibi, I, d. 3, p. 1, q. 1-2; ed. Vat. III, pp. 1-68, nn. 1-107.

Ibi, I, d. 8, p. 1, q. 3; ed. Vat. IV, pp. 222-223, nn. 138-139.

Ibi, I, d. 3, p. 1, q. 1-2; ed Vat. III, p. 18, n. 26.
} 
è il concetto insieme più perfetto o più semplice a noi possibile: questo infatti è più semplice che il concetto di ente buono e di ente vero, o di qualsiasi altro concetto simile, perché l'infinità non è un attributo o una passione dell'ente, ossia di ciò di cui si predica, ma esprime il modo intrinseco di essere di quell'entità; di modo che quando dico "ente infinito", non ho un concetto derivato quasi accidentalmente dall'ente o dalla passione, ma un concetto pertinente di per sé al soggetto esistente in un determinato grado di perfezione ossia dell'infinità.

Il concetto più semplice e più perfetto che la mente umana possa forgiare è dunque quello di "ens infinitum": è il più semplice, in quanto non è attributo dell'essere, ma un suo modo intrinseco; esso è caratterizzabile come "simpliciter simplex", e si distingue dagli altri concetti predicabili del "primum ens" ricavati astraendo dalle perfezioni delle creature. ${ }^{6}$ Scoto infatti distingue il "conceptus simplex, qui concipitur una intellectione et uno actu intelligendi", in "simpliciter simplex", ossia il concetto che non può essere ricondotto a concetti o nozioni anteriori, e il concetto "non simpliciter simplex", ossia il concetto che è suscettibile di definizione e quindi è risolvibile in altro o in concetti primi, come "uomo" è risolvibile in "animale" e "razionale".

La nozione di ente infinito è più semplice della nozione di soggetto e di qualche cosa ad esso attribuito, ove ciascun termine è formalmente distinto. Un modo intrinseco, quale l' "infinitas", non è formalmente distinto dal suo soggetto, ma gli appartiene per sé, laddove gli attributi sono "sicut additum".

La nozione di "ens infinitum" include virtualmente e in modo semplice tutte le perfezioni pure e ciascuna nel massimo grado possibile "sub ratione infiniti":

Probatur perfectio istius conceptus, tum quia iste conceptus, inter omnes nobis conceptibiles conceptus, virtualiter plura includit - sicut enim ens includit virtualiter verum et bonum in se, ita ens infinitum includit verum infinitum et bonum infinitum, et omnem "perfectionem simpliciter" sub ratione infiniti. ${ }^{8}$

L'ente infinito è la nozione più perfetta, in quanto include virtualmente la bontà infinita, la verità infinita e tutte le altre perfezioni che sono compatibili con l'infinitezza. Il contenuto virtuale, in questo caso, dal momento che non è formalmente identico al concetto, non può essere scoperto attraverso l'analisi della nozione di ente infinito. Scoto dunque, parlando di "ens infinitum" come del concetto più semplice, cerca di prospettare il caso di un'entità più grande di quella ottenibile con concetti sia semplici, sia complessi che possono essere definiti, ed è convinto che la compatibilità tra "infinito" ed "ente" è qualcosa per cui l'uomo possiede una sorta di evidenza psicologica intuitiva, una congenita aspirazione a un conoscere infinito e a un volere infinito.

Ibi, I, d. 3, p. 1, q. 2; ed. Vat. III, p. 40, n. 58.

Cfr. ibi, I, d. 2, p. 1, q. 2; ed. Vat. II, p. 142, n. 31.

Cfr. ibi e ID., Lectura I, d. 3, p. 1, q. 1-2; ed Vat. XVI, p. 250, n. 68.

Ordinatio I, d. 3, p. 1, q. 1-2; ed Vat. III, pp. 40-41, n. 59. Cfr. Lectura I, d. 3, p. 1, q. 1-2; ed. Vat. XVI, p. 244, nn. 50-53. 
Si tratta ora di chiarire il rapporto tra la nozione di infinito e gli altri trascendentali. Scoto divide i trascendentali dell' "ens inquantum ens" in due gruppi: "passiones simplices convertibiles" ("unum", "verum", "bonum") e "passiones entis disiunctae" ("unum" - "multa"; "idem" - "diversum"). Questi ultimi sono i predicati disgiuntivi, in quanto composti dalla disgiunzione di due predicati mutuamente esclusivi, quali "necessarium vel possibile", "infinitum vel finitum". Ciascun componente della coppia di predicati, singolarmente preso, non può essere predicato in modo conveniente di tutto l'ente, ma, se posti nella disgiunzione che ne evidenzia la reciproca incompossibilità, i due predicati coprono insieme l'intera estensione del concetto di ente (per il quale dunque sono compossibili).

Ciascuna di queste coppie opera una ripartizione nell'insieme delle entità, dato che ogni entità appartiene ad uno ed uno solo dei due sottoinsiemi definiti dai predicati in disgiunzione:

passiones disiunctae sunt transcendentes, et utrumque membrum illius disiuncti est trascendens quia neutrum determinat suum determinabile ad certum genus: et tamen unum membrum illius disiuncti formaliter est speciale, non conveniens nisi uni enti sicut necesse-esse in ista divisione "necesse-esse vel possibile-esse", et infinitum in ista divisione "finitum vel infinitum", et sic de aliis. ${ }^{9}$

Nel caso di "finitas vel infinitas" si assiste ad una peculiarità (quella dell' "infinitas") che, nella valenza più rigorosa di infinità intensiva, spetta solo a Dio. Mentre infatti le perfezioni divine, come la bontà e le altre "rationes formales", sono in qualche modo partecipabili dalle creature, l' "infinitas" che caratterizza l'essere e l'agire divino, eccede ogni partecipabilità e decide dell'inviolabile singolarità dell'essenza divina (1' "infinitas" è una perfezione singolare impartecipabile).

Nel suo Commento al Periermeneias, Scoto, riproponendo la questione della "infinitatio" dei termini trascendentali, si inserisce a pieno titolo in una lunga tradizione di pensiero che ha elaborato e sostenuto la tesi secondo cui "nomina infinita non possunt infinitari":

Ideo solet dici, et bene, quod ens et aliquid non possunt infinitari; quia nomen infinitum aliquid ponit, et non privatur per nomen, quod ponitur. ${ }^{10}$

L'infinitatio si ottiene aggiungendo la particella negativa "non" ad un nome indicante una realtà particolare (es. uomo), e perciò essa non può essere applicata ai nomi già di per sé infiniti o "omnia continentes" ("ens" o "aliquid"). Esistono dunque alcuni termini che sono significativi e nello stesso tempo di per sé infiniti, senza l'aggiunta della particella "non". I nomi trascendentali sono infiniti, perché non sono riconducibili ad un genere particolare, essendo piuttosto i principi analo-

\footnotetext{
Ordinatio I, d. 8, p. 1, q. 3; ed. Vat. IV, p. 207, n. 115. Cfr. A. GHISALBERTI, Ens infinitum e dimostrazione dell'esistenza di Dio in Duns Scoto, in: John Duns Scotus. Metaphysics and Ethics, ed. L. Honnefelder; R. Wood; M. Dreyer, Leiden, New York, Köln, 1996, pp. 415-434.

10 G. Duns Scoto, In Periermeneias op. II, q. IV; ed. Vivès I, pp. 590-591.
} 
gici di tutti i generi: "aliquid intentionale univocum, potest applicari rebus omnium generum".11

L'affermazione "aliquod ens est infinitum" trae la coerenza logica dalla natura della compossibilità dell'ente: se dal punto di vista del soggetto conoscente ("metaphysica nostra") si possono cogliere le nozioni trascendentali di "unum", "verum", "bonum", "ex ratione entis" è conoscibile l' "infinitas", quale trascendentale disgiuntivo. La natura dell'ente è segnata dalla compossibilità rispetto all'infinito. L'infinito è noto a partire dal tutto della compossibilità e dalle parti del tutto che sono anch'esse compossibili.

L'infinito è, per l'intelletto umano, un concetto astratto, al quale si arriva dalla nozione di finito; per astrazione, dalla nozione di "sommo" o di "più alto" e da una sorta di intuizione del dominio della potenza come di un tutto, si arriva al concetto di infinito: "coniungere intentionem summitatis intentioni entis vel boni et sic cognoscere summum ens vel bonum, et sic de infinito".

Questo tipo di astrazione conduce l'uomo ed una nozione che è massima in comprensione e minima in estensione, così minima da poter essere applicata solo ed esclusivamente a Dio. L'uomo congiunge l'ente e l'infinità ed arriva a pensare un ente infinito in perfezione e potenza, in quanto infinito è ciò che eccede qualsiasi finito, non solo perché eccede qualsiasi tipo di rapporto determinato, ma perché eccede tutti i tipi di rapporto immaginabili. L'infinito eccede pertanto ogni finito anche in relazione a qualsiasi misura o proporzione definita o definibile: nonostante la sua origine astratta, l' "infinitas" coincide con il costitutivo formale dell'essenza divina, più che con un suo attributo. Per definire l'infinito Duns Scoto si avvale della categoria della possibilità:

Voco autem hic infinitum quod quodcumque ens finitum datum vel possibile dari, excedit secundum omnem determinatam proportionem, acceptam vel acceptabilem. ${ }^{12}$

L'infinito è caratterizzato come ciò che eccede ogni ente finito attuale o possibile ed in riferimento a qualsiasi misurazione data o che possa darsi.

Si deve a Simo Knuuttila l'avere evidenziato l'importanza e la forza innovativa della posizione di Duns Scoto circa la teoria della modalità. Il paradigma della modalità prevalente nel mondo antico è quello statistico o della frequenza temporale della modalità, che si applica a enunciati temporalmente indefiniti.

Secondo Aristotele l'affermazione "A siede" è vera, ma sarà falsa dopo che $A$ si sarà alzato. I valori di verità riferiti alla modalità sono soggetti alla frequenza temporale, per cui si può dire che "se un enunciato vero ora, è vero tutte le volte che è proferito, esso è necessariamente vero. Se il suo valore di verità cambia nel tempo, esso è possibile. E se un enunciato è falso tutte le volte che è proferito, esso è impossibile". ${ }^{13}$

1 ID., Super Praedicamenta q. 3, n. 3; ed. Vivès I, p. 442.

2 ID., Reportatio I A 51; ed. in A. B. WolTER - M. MCCORD, Duns Scotus' Parisian proof for the existence of God, in "Franciscan Studies" 42 (1982), [248-321] p. 282.

13 S. KnUUTTILA, La critica di Duns Scoto allinterpretazione "statistica" della modalità, in AA.VV., Logica e linguaggio nel Medioevo, a cura di R. Fedriga e S. Puggioni, Milano 1993 (401-414), p. 402. 
Questa concezione della modalità è legata al principio di pienezza, in base al quale ogni possibilità genuina, per essere vera possibilità, deve verificarsi in un determinato momento del tempo. Duns Scoto si distacca da questo modello diacronico, secondo cui nessuna autentica possibilità può rimanere non realizzata nella successione temporale, ed istituisce un modello sincronico, in base al quale si ammette che qualcosa, che esiste o accade, possa essere o accadere in modo diverso nello stesso istante di tempo. Perciò la contingenza esprime la "possibilità" che si diano "simul" degli opposti. Questa possibilità è stabilita in rapporto ad un'azione causale che proceda attraverso intelligenza e volontà.

La stessa volontà umana è libera di fronte ad atti opposti, così come di fronte ad oggetti opposti. In riferimento agli oggetti opposti che mediante gli atti opposti essa può volere, la libertà umana esprime perfezione perché risulta detentrice di una duplice possibilità e contingenza: quella di volere successivamente degli oggetti opposti, e quella di volere contemporaneamente degli oggetti opposti, che però non possono essere in realtà scelti perché essa opera in modo successivo. Resta la perfezione della volontà che può volere (potenza "logica", ma non per questo irreale) simultaneamente tutto ciò che non è logicamente incompossibile. Ciò che determina l'impossibilità di qualcosa non è dunque la non-realizzazione nel mondo fattuale, bensì l'incompossibilità concettuale, la quale configura una contraddizione tra una possibilità pensata e la compossibilità di qualche cosa d'altro, parimenti pensato.

Se rileggiamo ora la nozione di infinità come ciò che eccede ogni ente finito, attuale o possibile, vediamo che con essa Scoto viene a dire che l'ente infinito eccede non solo ogni ente che in qualche momento di tempo si è realizzato, o si realizzerà, o si potrà realizzare, ma eccede anche ogni possibilità che non implichi incompossibilità, lasciando così spazio alla sola essenza divina. In questo modo è fugato ogni vincolo "ex parte rei" alla potenza di Dio, che E. Tempier aveva reiteratamente ravvisato nei continui legami della teologia aristotelica con la fisica e con l'unico mondo per Aristotele "realmente" possibile. D'altra parte, la metafisica riacquista in Scoto la sua specificità, stabilita dall'oggetto proprio (l'“ens inquantum ens"), al quale non si deve minimamente derogare nella dimostrazione dell' "ens infinitum".

\section{Infinità dell'essere e aspirazione all'infinito}

Un percorso determinante circa l'infinito è offerto dalla connessione tra sommo bene ed essere infinito. Nel De primo principio, argomentando l'infinità di Dio, il Dottore Sottile scrive: "Una sesta via per giungere alla conclusione dell'infinità può essere desunta dal fine. La nostra volontà può desiderare o amare qualcosa di più grande di qualisiasi fine limitato, come l'intelletto può, dal canto suo, conoscerlo. Sembra anzi che la volontà possieda un'inclinazione ad amare sommamente il Bene infinito. Infatti l'esistenza di un'inclinazione naturale nella volontà verso una cosa si arguisce dal fatto che la vuole prontamente e gioiosamente, pur non 
avendone l'abitudine. Ora, la volontà libera - come ci sembra di percepirla attraverso l'amore del Bene infinito - non riposa perfettamente che nel Bene sommo". ${ }^{14}$

La stessa considerazione è sviluppata nell'Ordinatio, dove è costruita come terza via per dimostrare l'infinità di Dio. L'esperienza interna dell'uomo, a parere di Duns Scoto, suffraga queste due constatazioni: la volontà umana, il cui oggetto è il bene, non si appaga mai nel possesso di un bene finito; il desiderio dell'uomo è sempre pronto ad "appetere et amare" qualcosa di maggiore, un bene più grande di qualsiasi bene finito dato. Inoltre, la volontà mostra la propria naturale inclinazione ad amare al massimo un bene infinito: l'inclinazione naturale della volontà verso qualche cosa è infatti evidenziata dal fatto che di sua iniziativa, senza un previo abito, vuole quella cosa "prompte et delectabiliter", ossia immediatamente e con appagamento del desiderio, e tale è l'inclinazione della volontà umana verso il bene infinito. Questi dati consentono di concludere non solo che l'uomo esperisce attualmente in sé il desiderio di amare un bene infinito, ma altresì che la volontà umana non sembra acquietarsi in modo perfetto in nessun altro bene. La conferma è data dal fatto che l'uomo odia il non-essere, ossia la natura razionale rifugge da tutto ciò che si configura come distruttivo dell'ordine ontologico: se il bene infinito risultasse qualcosa di impossibile e di assurdo, qualcosa di contrario all'oggetto del volere umano, la volontà lo odierebbe, ossia lo rifuggirebbe istintivamente. ${ }^{15}$

L'argomentazione di Duns Scoto mira a stabilire l'infinità come caratteristica di Dio, ossia di quell'essere trascendente che è stato precedentemente dimostrato come detentore di tre primalità o perfezioni nel sommo grado, in quanto causa efficiente prima, causa finale ultima e natura supremamente perfetta. La qualifica dell'infinità esprime per il Dottor Sottile il vertice della perfezione formale di Dio; sappiamo già che, per l'uomo, l'infinità è il concetto più elevato che possa avere di Dio in questa vita, e perciò il nostro maestro si era premurato di mostrare preliminarmente la non ripugnanza dell'infinità all'ente: "enti non repugnat infinitas". Non c'è contraddizione tra il concetto di ente e il concetto di infinito, perché l'intelletto non prova alcuna ripugnanza nel pensare qualcosa di infinito, lo vede anzi come l'intelligibile più perfetto. Del tutto analogamente l'aspirazione della volontà dell'uomo a un bene infinito non si presenta come la passione inutile o irrazionale di un soggetto inappagato dai risultati delle proprie azioni; essa è calata in un fondo di razionalità.

L'intuizione come la fruizione diretta di un ente-bene caratterizzato dall'infinità non sono tuttavia garantite all'intelletto finito e alla volontà finita dell'uomo viatore: l'infinito è "obiectum naturale" di un intelletto e di una volontà naturalmente infiniti. Da ciò si ricava una prima considerazione, relativa a quella

4 DUNS SCOTO, Il primo principio degli esseri, trad. di P. Scapin, Padova 1973, p. 246.

"Voluntas nostra omni finito aliquid maius potest appetere et amare. [...] Nam inde arguitur inclinatio naturalis ad aliquid in voluntate, quia ex se, sine habitu, prompte et delectabiliter vult illud voluntas libera; ita videtur quod experimur actu amandi bonum infinitum, immo non videtur voluntas in alio perfecte quietari. Et quomodo non illud naturaliter odiret si esset oppositum sui obiecti, sicut naturaliter odit non-esse?" (DUNS SCOTO, Ordinatio I, dist. 2, p. 1, q. 1-2; ed. Vat. II, pp. 205-206, n. 130). 
che potremmo chiamare l'etica della conoscenza: affermando l'esistenza di un essere infinito nell'ordine delle conoscenze, si afferma contemporaneamente l'esistenza di un ambito di conoscenze eccedente l'orizzonte delle conoscenze intellettive dell'uomo; l'intelletto infinito di Dio istituisce un sapere transmetafisico, ossia si deve ammettere che all'affermazione dell'esistenza dell'infinito consegue l'affermazione dell'ordine delle conoscenze proprio dell'essere infinito, ulteriore a ogni sapere metafisico dell'intelletto umano, e che nel linguaggio di Duns Scoto è definito la "theologia in se", naturalmente intenzionata dall'intelletto divino e alla quale l'uomo ha accesso solo se una rivelazione positiva gliene offre dei contenuti articolati e resi comprensibili dalle forme del linguaggio umano. La rivelazione appare così in una prospettiva che dice la compatibilità e l'intrinseca coerenza tra l'ordine delle conoscenze dell'intelletto umano e l'ordine delle verità rivelate, proprio perché la dimostrazione dell'esistenza dell'infinito comporta l'ammissione dell'esistenza di un sapere infinito, per sua natura sottratto all'intelletto del metafisico.

La rivelazione di alcuni contenuti di questo sapere infinito assume perciò i connotati di coerente supporto alla natura dell'intelletto umano, che non dispone in proprio di possibilità alcuna di accedere per altra via alla conoscenza di quell'essere infinito, di cui ha peraltro dimostrato l'esistenza. L'analisi dell'infinito ci riporta cioè all'insistenza su di un aspetto della rivelazione, per cui essa risulta perfezionamento delle potenze naturali, conoscitive e volitive dell'uomo, pur senza derogare in nulla al primato dell'iniziativa divina nel rivelare e alla sua gratuità. Come magistralmente si esprime Paul Vignaux:

Ponendo al di là di sé una "deità" - deitas sub ratione deitatis - conoscibile solo per divina rivelazione, il concetto scotista di infinito mette la teologia naturale in una situazione in cui non potrebbe entrare, come temerà Karl Barth, in concorrenza con la parola di Dio su se stesso. Nel medesimo tempo la specificità della Rivelazione e della teologia libera il teologo e ogni credente che riflette da qualunque complesso di inferiorità intellettuale. ${ }^{16}$

Una seconda e ultima considerazione emerge dalla connessione esplicita operata da Duns Scoto parlando dell' "infinitas Dei", tra la natura dell'intelletto divino che deve avere simultaneamente presente un'infinità di oggetti, dall'eternità, distintamente e indipendentemente dalla loro esistenza, e la volontà onnipotente di Dio o potenza causale atta a creare una infinità di cose, ossia la perfezione dell'efficienza propria della causa di tutto l'essere attuale e possibile. L'aspirazione umana ad un bene infinito è risultata non velleitaria e non contraddittoria proprio perché l'infinità non ripugna all'intelletto e al volere; non siamo dunque in una prospettiva di "volontarismo", non siamo di fronte a una prevaricazione che attribuisce alla volontà totale autonomia rispetto all'intelletto, come spesso la storiografia della prima metà del Novecento ha sostenuto in riferimento a Duns Scoto. Il percorso, che ha condotto all'affermazione dell' "ens infinitum" e al riconoscimento

P. VIGNAUX, La filosofia nel Medioevo, Bari, Laterza, 1990, p. 180. 
della intrinseca validità dell'aspirazione dell'uomo all'infinito, mette in risalto lo stretto rapporto tra essere e bene, tra intelligenza e volontà, che la libertà della volontà non potrà mai alterare $\mathrm{o}$ sopprimere, perché non potrà mai decidere di annullare la propria natura più intima e costitutiva, ossia la strutturale capacità della volontà dell'uomo di amare l'oggetto più amabile, di volere il bene più grande, di desiderare cioè la fruizione di un bene infinito.

Dall'analisi dei tratti caratteristici del volto filosofico di Dio come ente infinito, Duns Scoto riesce a dedurre i lineamenti e i connotati del volto dell'uomo; l'infinito svela il finito, rivela i tratti più reconditi e significativi di un soggetto che non solo è capace di conoscere e di amare gli enti finiti o i beni limitati, ma che è strutturalmente aperto alla totalità dell'essere e del bene, al punto che solo un abbraccio con l'infinito può saziare ogni suo desiderio. 